but we stated that, according to our results, IFN- $\gamma$ based assays could reduce unnecessary chemoprophylaxis in non- $M$. tuberculosis infected children. In fact, BAKIR et al. [11] in a recent study concluded that a positive IFN- $\gamma$ based assay result predicted the development of active TB as well as the TST, allowing more focused preventive therapy to fewer contacts.

In conclusion, we believe our results provide enough evidence that previous NTM sensitisation induces false-positive results in the TST for diagnosing LTBI; but, we also strongly agree with TEBRUeGge et al. that additional studies are needed in order to clarify different issues related to the discordant IFN- $\gamma$ based assay results, and to assess the real utility in the management and benefit of a child population.

I. Latorre ${ }^{*, \tau,+}$, M. De Souza-Galvão ${ }^{\uparrow, \S}$, J. Ruiz-Manzano ${ }^{\#, \uparrow,+}$, A. Lacoma ${ }^{*,+,+}$, C. Prat ${ }^{*,+,+}$, N. Altet ${ }^{\S}$, V. Ausina ${ }^{*,+,+}$ and J. Domínguez ${ }^{*,},++$

*Servei de Microbiologia, and "Servei de Pneumologia, Hospital Universitari "Germans Trias i Pujol", Fundació Institut d'Investigació en Ciències de la Salut Germans Trias i Pujol, ${ }^{+}$Ciber Enfermedades Respiratorias, Instituto de Salud Carlos III, Badalona, "Universitat Autònoma de Barcelona, Bellaterra, and ${ }^{\S}$ Unidad de Prevención y Control de la Tuberculosis de Barcelona, Barcelona, Spain.

Correspondence: J. Domínguez, Servei de Microbiologia, Fundació Institut d'Investigació en Ciències de la Salut "Germans Trias i Pujol", Carretera del Canyet s/n, 08916 Badalona, Barcelona. Spain. E-mail: jadomb@gmail.com

Statement of Interest: A statement of interest for J. Domínguez can be found at www.erj.ersjournals.com/misc/statements.dtl

\section{REFERENCES}

1 Latorre I, De Souza-Galvao M, Ruiz-Manzano J, et al. Evaluating the non-tuberculous mycobacteria effect in the tuberculosis infection diagnosis. Eur Respir J 2010; 35: 338-342.

2 Domínguez J, Ruiz-Manzano J, De Souza-Galvao M, et al. Comparison of two commercially available gamma interferon blood tests for immunodiagnosis of tuberculosis. Clin Vaccine Immunol 2008; 15: 168-171.

3 Domínguez J, Latorre I, Altet N, et al. Interferon-gamma-release assays to diagnose TB infection in immunocompromised individual. Expert Rev Respir Med 2009; 3: 309-327.

4 Connell TG, Ritz N, Paxton GA, et al. A three-way comparison of tuberculin skin testing, QuantiFERON-TB gold and T-SPOT.TB in children. PLOS ONE 2008; 3: e2624.

5 Lein AD, von Reyn CF, Ravn P, et al. Cellular immune responses to ESAT-6 discriminate between patients with pulmonary disease due to Mycobacterium avium complex and those with pulmonary disease due to Mycobacterium tuberculosis. Clin Diagn Lab Immunol 1999; 6: 606-609.

6 Nyboe J. Interpretation of tuberculosis infection age curves. Bull World Health Organ 1957; 17: 319-339.

7 Ruiz-Manzano J, Blanquer R, Calpe JL, et al. SEPAR Guidelines. Diagnostic and treatment of tuberculosis. Arch Bronconeumol 2008; 44: 551-566.

8 Detjen AK, Keil T, Roll S, et al. Interferon-gamma release assays improve the diagnosis of tuberculosis and nontuberculous mycobacterial disease in children in a country with a low incidence of tuberculosis. Clin Infect Dis 2007; 45: 322-328.

9 Haimi-Cohen Y, Zeharia A, Mimouni M, et al. Skin indurations in response to tuberculin testing in patients with nontuberculous mycobacterial lymphadenitis. Clin Infect Dis 2001; 33: 1786-1788.

10 Lalvani A, Millington KA. T cell-based diagnosis of childhood tuberculosis infection. Curr Opin Infect Dis 2007; 20: 264-271.

11 Bakir M, Millington KA, Soysal A, et al. Prognostic value of a Tcell-based, interferon-gamma biomarker in children with tuberculosis contact. Ann Intern Med 2008; 149: 777-787.

DOI: $10.1183 / 09031936.00033510$

\title{
Complete smoking cessation is beneficial in older and more advanced COPD patients
}

\section{To the Editors:}

We read with great interest the article by TASHKIN et al. [1] in a recent issue of the European Respiratory Journal evaluating effects of smoking status on long-term responses to maintenance bronchodilator therapy in the Understanding Potential Long-term Impacts on Function with Tiotropium (UPLIFT ${ }_{\circledR}$ ) trial. The UPLIFT ${ }_{\circledR}$ trial [2] is a recent investigation in a long series of clinical trials assessing, among other things, the effects of different drugs on long-term forced expiratory volume in $1 \mathrm{~s}$ (FEV1) decline in patients with COPD, a "holy grail" of the pulmonological community.
As tobacco smoking is the most frequent risk factor for COPD, researchers in the UPLIFT ${ }_{\circledast}$ trial paid the utmost attention to smoking status of the investigated cohort. They registered smoking status at inclusion, offered smoking cessation to every smoking patient before entry and checked smoking status at each follow-up visit during the $4 \mathrm{yrs}$ of study. Study participants were classified into three subgroups: continuing current smokers (CS), continuing ex-smokers (CE) and intermittent smokers (IS).

The authors concentrate on analysis of effects of tiotropium in relation to smoking status on bronchodilation, exacerbation 
rate, quality of life and mortality. They acknowledge that the rate of FEV1 decline was highest in CS, and lowest in CE in both tiotropium and placebo (other treatments) subgroups. We thank the authors for highlighting this finding in their abstract, and feel that this particular finding deserves some comment.

The authors compare their study to the Lung Health Study (LHS) [3] in respect to effect of smoking status on the rate of FEV1 decline. The LHS and UPLIFT ${ }^{\circledR}$ studies have many similarities: large study groups, smoking cessation programme at entry, long-term follow-up and three smoking status subgroups. However, it is most interesting that the UPLIFT ${ }_{\circledR}$ trial is complementary to LHS [4]. LHS participants were younger, with a mean age 48.5 yrs versus $61-66$ yrs in $\mathrm{UPLIFT}_{\circledast}$. The majority of LHS participants were in the mild/moderate stage of COPD (mean FEV1 at $78 \%$ of predicted, range 55-90\%), whereas no mild stage patients were included in the UPLIFT ${ }_{\circledR}$ trial (mean FEV1 at $40 \%$ pred, range $20-70 \%$ ); $96 \%$ of participants were in the moderate or severe stage.

Interestingly, mean FEV1 decline per year in the first 5 yrs of follow-up in LHS continuing smokers was $62 \mathrm{~mL} \cdot \mathrm{yr}^{-1}$ versus $59 \mathrm{~mL} \cdot \mathrm{yr}^{-1}$ in the UPLIFT ${ }_{\circledast}$ CS subgroup. Continuing exsmokers in LHS lost $31 \mathrm{~mL} \cdot \mathrm{yr}^{-1}$ versus $33 \mathrm{~mL} \cdot \mathrm{yr}^{-1}$ in the $\mathrm{UPLIFT}_{\circledast}$ trial; almost superimposed figures. Thus, smoking cessation was highly effective across the entire spectrum of COPD severity.

The UPLIFT ${ }_{\circledR}$ trial demonstrated that complete smoking cessation results in clinically significant reduction in FEV1 decline in COPD patients with more advanced stages of the disease, irrespective of treatment. A useful future objective for the authors would be to perform statistical analysis of differences in FEV1 decline in the three subgroups of smoking status.

This new piece of evidence resolves reservations that spectacular effects of smoking cessation on FEV1 decline demonstrated in the LHS may be limited to COPD patients with mild forms of the disease [5]. Another important message from the TASHKIN et al. [1] study is that only CE patients had improved survival, irrespective of treatment modality, another finding in conjunction with the results of LHS after 14.5 yrs follow-up [6]. The majority of COPD patients have cardiovascular comorbidity. Successful smoking cessation would also reduce risk for myocardial infarction or stroke.

Complete smoking cessation remains a much more effective method to stop accelerated FEV1 decline in COPD than any pharmacological agent tried so far.

The results of the UPLIFT ${ }_{\circledR}$ trial support the idea of early detection of COPD by high-risk population screening [7] or a case finding method [8]. Early diagnosis gives a unique opportunity to target that group with the full array of antismoking initiatives. All available resources should be applied to help smokers with COPD to quit smoking as a first-line treatment. Spirometry testing of smokers combined with smoking cessation clinics increase smoking cessation rate [9]. Our observational study demonstrating that $17 \%$ of smokers with spirometric signs of airflow obstruction stop smoking has recently been confirmed by large randomised controlled investigations [10]. We strongly believe that early detection of COPD combined with primary and secondary prevention measures are the best methods to reduce the burden of the disease [11].

\section{J. Zielinski, M. Bednarek and D. Gorecka}

Dept of Respiratory Medicine, National Research Institute of Tuberculosis and Lung Diseases, Warsaw, Poland.

Correspondence: J. Zielinski, Dept of Respiratory Medicine, National Research Institute of Tuberculosis and Lung Diseases, Warsaw, Poland. E-mail: j.zielinski@igichp.edu.pl

Statement of Interest: None declared.

\section{REFERENCES}

1 Tashkin DP, Celli B, Kesten S, et al. Long-term efficacy of tiotropium in relation to smoking status in the UPLIFT trial. Eur Respir J 2010; 35: 287-294.

2 Tashkin DP, Celli B, Senn S, et al. A 4-year trial of tiotropium in chronic obstructive pulmonary disease. $N$ Engl J Med 2008; 359: 1543-1554.

3 Anthonisen NR, Connett JE, Kiley JP, et al. Effects of smoking intervention and the use of an inhaled anticholinergic bronchodilator on the rate of decline of FEV1. The Lung Health Study. JAMA 1994; 272: 1497-1505.

4 Scanlon PD, Connett JE, Waller LA, et al. Smoking cessation and lung function in mild-to-moderate chronic obstructive pulmonary disease. The Lung Health Study. Am J Respir Crit Care Med 2000; 161: 381-390.

5 Pride NB. Smoking cessation: effects on symptoms, spirometry and future trends in COPD. Thorax 2001; 56: Suppl. 2, ii7-10.

6 Anthonisen NR, Skeans MA, Wise RA, et al. The effects of a smoking cessation intervention on 14.5-year mortality: a randomized clinical trial. Ann Intern Med 2005; 142: 233-239.

7 Zielinski J, Bednarek M, Gorecka D, et al. National Program of early detection and prevention of COPD in the years 2000-2002. Pneumonol Alergol Pol 2005; 73: 116-121.

8 Buffels J, Degryse J, Heyrman J, et al. Office spirometry significantly improves early detection of COPD in general practice: the DIDASCO Study. Chest 2004; 125: 1394-1399.

9 Bednarek M, Gorecka D, Wielgomas J, et al. Smokers with airway obstruction are more likely to quit smoking. Thorax 2006; 61: 869-873.

10 Parkes G, Greenhalgh T, Griffin M, et al. Effect on smoking quit rate of telling patients their lung age: the Step2quit randomised controlled trial. BMJ 2008; 336: 598-600.

11 Soriano JB, Zielinski J, Price D. Screening for and early detection of chronic obstructive pulmonary disease. Lancet 2009; 374: 721-732.

DOI: $10.1183 / 09031936.00027810$

From the authors:

We thank J. Zielinski and co-workers for their comments and concur with their conclusion that the significant and substantial benefits of complete smoking cessation and sustained abstinence with respect to reduction in the annual rate of decline in forced expiratory volume in $1 \mathrm{~s}$ (FEV1) that were noted in the mostly middle-aged participants in the landmark Lung Health Study [1-3] were similarly observed in older patients with more advanced chronic obstructive pulmonary disease (COPD), 